\title{
Medication errors safety signals COVID-19-related Moroccan PV database
}

\author{
HoudaSefiani ${ }^{1,3}$, Ghita Benabdallah ${ }^{2}$,Amina Tebaa ${ }^{2}$, Rachida Soulaymani Bencheikh $h^{2,3}$ \\ ${ }^{1}$ Research Laboratory Innovation \& Information System, EuRSED Vienne France. \\ ${ }^{1}$ Centre antipoison et de pharmacovigilance, WHO-CC Rabat Morocco \\ ${ }^{3}$ Faculté de médecine et de pharmacie de Rabat, Morocco
}

\begin{abstract}
On March 23, 2020, the Moroccan Ministry of Health issued a circular authorizing the off label use of hydroxychloroquine or chloroquine in combination with azythromycin as first-line treatment for all COVID-19 patients. The aim of this study is to detect, assess and manage safety signals from individual cardiovascular adverse events (AE) reported. Methods: During the COVID19 pandemic, pharmacovigilance surveillance was based on spontaneous targeted notification in all health facilities. Newsletters with a suitable reporting form were sent and reporting of AEs was mandatory. The AEs collected were analyzed for causality assessment using the WHO method and the Preventavility method was used to detect any preventable ADR. Safety signals were detected and analyzed using a qualitative pharmacovigilance method. Results: As of December 31, 2020, a total of 527 adverse events have been reported to the NPC related to Hydroxychloroquine or Chloroquine among the Covid 19 patients. We received 09 cases of preventable AEs related to medication error including one drug interaction for 3 cases, dose errors for 5 cases and one case with both errors, these cases have been validated ad managed as safety signals. Discussion and conclusion: The detected signal concerned the QT prolongation, hypokalaemia and arrythmias, due to high dose of azithromycin and to drug interactions with hypokalaemic drugs. The signal was analyzed and validated during the technical committee, and recommendations were established to avoid the occurrence of similar cases.
\end{abstract}

\section{Introduction :}

The outbreak of coronavirus disease COVID-19 has created a global health crisis and on March $11^{\text {th }} 2020$, the WHO declared the COVID-19 as a pandemic [1]. Since then Countries around the globe have strenghtened their efforts to improve their surveillance system to quickly diagnose cases and to find an effective and safe way to manage and treat COVID 19 patients.

There is a long process that a new product has to go through before it can be used as drug to treat human deseases. To face the health crisis some countries have turned to existing drugs with potential therapeutic action, despite the absence of conclusive clinical trials for this use [2].

Chloroquine and hydroxychloroquine are approved for other uses, and their safety profiles have been well established. Furthermore, those drugs has been demonstrated to have an anti-SARS-CoV activity in vitro [3].

On March 23, 2020, The Moroccan ministry of health issued an circulaire and allow hydroxychloroquine sulphate or chloroquine sulphate to be used in association with azythromycin as first line treatment for all COVID-19 patients in hospital [4].

To support the implementation of the Covid 19 treatment protocol and to insure the rational use of drugs and patient safety, the Centre anti poison et de pharmacovigilance du Maroc (CAPM) has developed documents with recommendations for Health care professionals regarding the safe use of those drugs. In addition the CAPM launched atargeted surveillance to collect adverse event observed with patients in all health care facilities dedicated to COVID19 in the country to be able to react quickly to any safety signal detected.

Indeed, the performance of any pharmacovigilance system is measured by its ability to detect signals and manage alerts to ensure patient safety. Typically, the quantitative methods available are applicable for large databases containing more than 500 cases [5]. In the current situation, the number of cases collected is low and the best way to detect safety problem is to use the qualitative methods.

The aims of this study is to detect, evaluate, and manage safety signals arising from individual case safety reports (ICSR) with cardic ADRs reported with the use of HCQ /CQ and Azithromycine for COVID 19 treatment in Morocco.

\section{Methods}

ICSR reporting ADRsthe use of chloroquine or Hydroxychloroquine with azithromycin were collected using an adapted reporting form with specific items related to the Covid 19 disease [Annexe1].

ICSRswere received at the CAPM and analyzed for causality assessment using WHO causality assessment method[6], pharmacological plausibility was evaluated by compatible mechanism ofoccurence and compatible

\footnotetext{
${ }^{\text {*Corresponding author: hsefiani@gmail.com }}$
} 
time-to-onset. Adverse events were validated and a score was associated with each combination ADR/Drug. P method criteria were used to detect a preventable ADR [7].

After a first expertise by the pharmacovigilant, all cases were shared to the international database vigibase ${ }^{\circledR}$ the World Health Organization pharmacovigilance database. Qualitative method to detect and manage signals was applied. This method with integrated approach runs into 6steps:

1. Identification of signal :Cases with ADRs Classified according to the Medical Dictionary for Regulatory Activities MedDRA [8]in system organ class (SOC) 'Cardiac Disorders' and 'electrocardiogram abnormalities' included in the SOC 'Investigation' were extracted and the aggregate data was analyzed to extract clusters responding to signal definition in pharmacovigilance.

2. Cluster investigation: Each case within the clusters identified was reviewed by pharmacovigilants to complete the missing data required for investigation. Narrative of each case was evaluated. The aggregate data was deeply analyzed regarding patientscharachteristics (age, sexe), reported ADRs, comcomitant comorbidities, concomitant drugs and Circumstances of occurence to detect common risk factors or contributing factors.

3. Root cause analysis : signals arising were analyzed using the Ishikawa Fishbone diagram [9] to identify proximal causes and contributing factors.

4. Review databases and published data :previous similar cases were searched in local and internationalpharmacovigilance databases such as Vigibase, EudraVigilance and the FDA AERs. similar signals were searched in published data during Covid19 from NRAs and health authoritieswebsites and scientific literature.

5. Risk minimisationaction: adapted actions were proposed by the national technical committee and implemented after validation.

6. Assessment of implementedactions :adapted measurement of actions was implemented to assess that identified risk has been minimized (direct outcome)

\section{Results}

In a context of health crisis due to the spread of the virus SARS-CoV-2, responsible for the infectious disease Covid-19, qualified as a pandemic by the World Health Organization, the Ministry of Health in Morocco, in addition to preventive measures to limit the spread, has established a scientific advisory technical committee which adopted on March 21, 2020 recommendations for the management of patients confirmed positive. Curative treatment for positive tested patients have been put in place and given to all patient with or without symptoms. The first line protocoleincluded Chloroquine sulfate (Nivaquine ${ }^{\circledR} 100 \mathrm{mg}$ ) $500 \mathrm{mg}$ 2twice a day during 10 days (1000 mg per day) or Hydroxychloroquine sulfate
(Plaquénil $\left.{ }^{2} 200 \mathrm{mg}\right) 200 \mathrm{mg} \quad 3$ time aday during 10 days(600 mg per day) with Azithromycin $500 \mathrm{mg}$ the first day and $250 \mathrm{mg}$ per day during 6 following days.

Up to 31 December2020, a total of 527 ADRs were reported to CAPM with the use of chloroquine sulfate or Hydroxychrloquine sulfatewith Azithromycine in covid 19 patients, 283 of which associated with hydroxychloroquine sulfate $(53,7 \%)$. The majority of these reports $(78,7 \%)$ had been considered as "non serious" by the original reporter according to WHO serioussnesscriterias $[10,11], 54,5 \%$ of patient were female and sexe ratio $\mathrm{M} / \mathrm{F}$ was $0,74.48,2 \%$ of patients were aged between 18 and 44 years.

The included MedDRAterms: Cardiac disorders and ECG abnormalities were reported in 76 ICSRs as adverse drug reactions associated with the use of hydroxychloroquine or chloroquine and azithromycin in COVID-19 witch represented 14,42\% of total cases, 9 of these cases were classified as preventable. Drug-drug interaction was detected for 3 patients who took Hydroxychloroquine with Hypokaliemiant Diuretics and presented QT prolongation due to hypokaliemia. Dose errors were detected for 5 cases who took $500 \mathrm{mg}$ of Azithromycine every day instead of $250 \mathrm{mg}$ from the $2^{\text {nd }}$ to the 7 th day of the treatment. For one case both errors were reported[Table 1].

Table 1 : Patients characteristics and Analysis results

\begin{tabular}{|c|c|}
\hline \multicolumn{2}{|c|}{ Cases details : PreventableADRs } \\
\hline Patients age & $32-73$ ans \\
\hline Gender & 6 Males, 3 Females \\
\hline $\begin{array}{l}\text { CovidStatut : PCR + and } \\
\text { symptoms }\end{array}$ & $\begin{array}{l}\text { 9: PCR positive } \\
\text { 5: Covidsymptoms }\end{array}$ \\
\hline Concomitant diseases & $\begin{array}{l}4 \text { patients : Diabetis, HTA, } \\
\text { Brest cancer }\end{array}$ \\
\hline ReportedADRs: & $\begin{array}{l}\text { Extrasystoles, QT prolonged, } \\
\text { Torsades de pointe }\end{array}$ \\
\hline $\begin{array}{l}\text { WHO-UMC } \\
\text { causalityassessment }\end{array}$ & $\begin{array}{l}5 \text { probable } \\
4 \text { possible }\end{array}$ \\
\hline P méthod & $\begin{array}{l}\text { 9 Preventable ADR } \\
\text { - } 5 \text { dose errors } \\
\text { - } \quad 3 \text { drug-drug interaction } \\
\text { (Furosemid, } \\
\quad \text { hydroxychlorothiazid) } \\
\text { - } 1 \text { case : botherrors }\end{array}$ \\
\hline
\end{tabular}

The qualitative approach applied in the CAPM, allowed the early detection of 2 signals validated into alerts.

We performed a specific assessment of these preventable cardiac adverse drug reactions using the Ishikawa diagram to understand the root cause of occurrence of this errors. The analysis have shown that the occurrence or the detected errors are due to multiple contributong factors [ Figure 1]. 


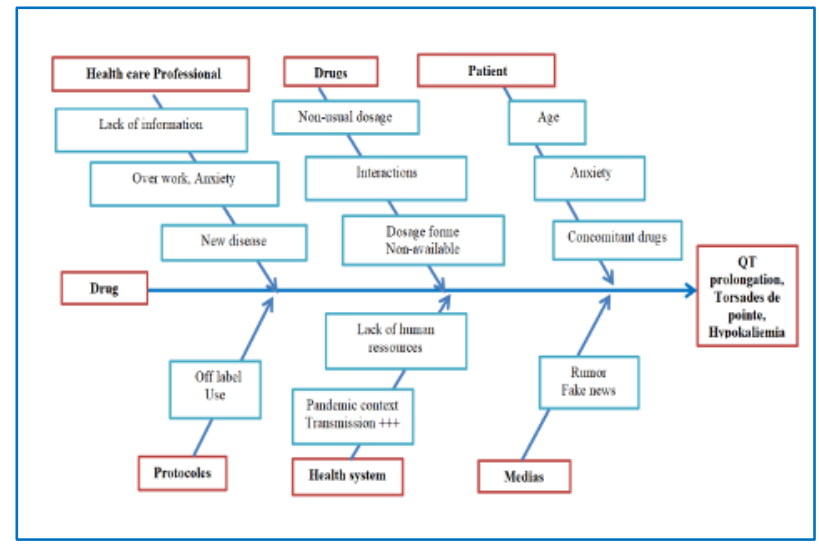

Figure 1: Root Causes analysis, contributing factors Ishikawa diagram

For the three cases with drug-drug interactions, the root causes detected was mainly the lack of knowledge of the risks of drug interactions on the junior doctors who were prescribing several drugs with a high torsadogenic risk. In addition, the information of the patients' long-term treatments was not be collected before the introduction of the anti-covid treatment.

Root cause analysis of the doses errors identified three contributing factors : the specific regimen (once daily) of Azithromycin, the Non-availability of the appropriate dosage form $(250 \mathrm{mg})$ and particularly the patient's lack of understanding of the protocol.

The databases and litterature review have shown that the dose errors with azithromycine are common and are due due to the non usual dosage of this antiinfective drug.

The situations with ADRs related to drug interactions errors with hydroxychloroquine/chloroquine are not reported.

After assessment during the technical committee, precentive action were proposed :

The fisrt action was regulotory, the drug regulatory authority was in charge to send a «Dear Doctor Letter » to all health care professionals containing:

- Comprehensive list of contributing factors that may increase the risk of cardiovascular adverse events

- List of torsadogenic, hypokalemic, and enzyme inhibiting drugs

- Preventive actions to be taken before treatment and corrective actions

- List of contraindications

- Obligation to do a deep patient interrogation to detect risk factors before starting the treatment

- Modalities of drug intake and dosage to avoid preventable adverse events

- Messages adapted to the patient to explain in a simple and precise way the doses and the rhythm of taking for each drug
Communication actions around the risk near HCPs and patient were the second part of recommended actions :

- Raising awareness among HCPs through scientific journals, social networks and webinars (to be organized regularly) on $\mathrm{HCQ} / \mathrm{CQ}$ and Azithromycin-induced hypokalemia

- Proposing Covid 19 patient management recommendations to be implemented and applied before the start of treatment, during treatment and after treatment in order to ensure patient safety and to prevent the occurrence of some ADRs

- Public education and information about the risk of fake news and misinformation that can endanger the public

- Communication to the public and patients via social media regarding the risks and the non compliance of the treatment.

The third part of recommended actions concerned health strategies and protocoles, thus were proposed regular updates of therapeutic and surveillance protocols based on epidemiological advances and results of harmacovigilance surveillance, this update should contain :

- Comprehensivelist of drug interactions

- Pre-therapeuticinterrogation and tests

- Protocole of monitoring and follow-up

In addition, the committee asked the pharmaceutical industries that market azithromycin to market the 250 $\mathrm{Mg}$ form adapted to the Covid 19 treatment protocol adopted by the ministry of health.

In all correspondances, the ministry of health and the CAPM made healthcare professionals aware of the importance to report all adverse events observed, including asymptomatic medication errors, to the Moroccan Center for Pharmacovigilance on the official CAPM reporting form.

The monitoring of adverse reactions and the analysis of the received cases after the actions undertaken allows the assessment of the actions impact. In fact, from the date of implementation of actions, no new case of adverse reaction related to dose errors or drug interactions has been reported after.

\section{Discussion}

Chloroquine sulfate (Nivaquine ${ }^{\circledR} \quad 100 \mathrm{mg}$ ) and Hydroxychloroquine sulfate (Plaquénil ${ }^{\circledR 2} 20 \mathrm{mg}$ ) are two drugs that have been used for over 70 years and have well established safety profiles [12].

They are indicated in the treatment, prevention of malaria and in the treatment of Rheumatoid Arthritis, Disseminated and Discoid Lupus Erythematosus, and Lucitis. Hydroxychloroquine is included in the WHO essential medicines list updated in 2019. 
Cardiac disorders are labeled with Chloroquine sulfate (Nivaquine ${ }^{\circledR} 100 \mathrm{mg}$ ) and Hydroxychloroquine sulfate (Plaquénil $\AA 200 \mathrm{mg}$ ), these are generally modifications of the electrocardiogram (altered $\mathrm{T}$ waves and lengthening of the QT interval, torsades de pointes), these disorders are dose dependent $[12,13]$.

Combination with azithromycin that can prolong the QT interval puts the patient at an additional risk of QT prolongation and torsade de pointes.

These expected Cardiac disorders and ECG abnormalities could represent a signal if the combination with Chloroquine/hydroxychloroquine, azithromycin, is assessed using an indicator value for disproportionate Bayesian reporting that compares observed and expected values to find a statistical association.

The signal in Pharmacovigilance is defined as "Reported information on a possible causal relationship between an adverse event and a drug, the relationship being previously unknown or incompletely documented" the quantitative signal detection using reporting odds ratio (ROR) or information component (IC) as indicator was not applicable in our system regarding the low number or reported cases [14].

The qualitative method allows the early detection of signals that are not detected by the quantitative methods that requires large databases. Otherwise the method allows the signal analysis of detected cluster of cases to detect the circunstances of occurrence.

The first signal detectedin our study was related a preventable ADR due to durg drug interaction for wich the root cause analysis have shown that it's due to the lack of knowledge regarding this risk. Indeed, the Covid19 patients could be with multiple comorbidities and simultaneously treated with other medications with cardiovascular risk like hypokalemic diuretics for hypertension. The cardiac toxicity induced by Hydroxychloroquine is dose-dependent occuringby inhibition of hERG potassium channels leading to risk of T-wave impairment, QTc prolongation and torsades de pointes. All this ADRs are labeled and well documented with known risk factors as hypokalemia, combination with azithromycin or any other hypokalemic, torsadogenicdrugs and the Covid disease itself.

To avoid similar errors, and to ensure rational use of drugs and patient safety, CAPM has issued an information letter to all health professionals to draws their attention to this type of error and recommends to perform a deep questioner of patient before starting the Covid 19 treatment and to change a long term therapy if necessary.In addition, blood tests and electrocardiograms at 24-hour intervals were recommended for all patients undergoing treatment in order to detect cardiac disorders at an early stage and to manage them rapidly.

Furthermore, the CAPM asks health care professionals to educate patients and the general public on the importance of respecting prescriptions and on the risks linked to self-medication, the irrational use of drugs and plants.

The second signal was related to dose errors of azithromycine. Dose errors related to the use of azithromycin are frequently reported in Morocco due to the fact that the dosage of one tablet $500 \mathrm{mg}$ per day is not usual for an antibiotic treatment. Within the the Covid19 protocol, taking one tablet $500 \mathrm{mg}$ on the first day and half a tablet $250 \mathrm{mg}$ from the second to the seventh day led to errors in the patients.

The automated quantitative analysis of signals in our database did not detect these signals related to medication errors.

\section{Conclusion}

"Off-label' use of hydroxychloroquine or chloroquine with azithromycin in COVID-19 deseaseincreases the risk ofcardiac ADRs in patients, some of them were detected as preventable due to medication errors. Even if these drugs are perceived as familiar, they areused in patients with added risk factors caused by infection or other comorbidities. The harm due to medication errors is preventable if cluters are detected early and adapted minimization actions are put in place to avoid their occurrence and to ensure patient safety.

\section{References}

1. World Health Organization. Who DirectorGeneral's Opening Remarks at the Media Briefing on Covid-19-11March 2020. Available online: https://www.who.int/dg/speeches/detail/whodirector-general-s-opening-remarks-at-the-mediabriefing-on-covid-19---11-march-2020 (accessed on 12 June 2021)

2. Wu, Z.; McGoogan, J.M. Characteristics of and important lessons from the coronavirus disease 2019(COVID-19) outbreak in China: Summary of a report of 72314 cases from the chinese center for diseasecontrol and prevention.JAMA (2020)

3. Das, Saibal et al. "An Updated Systematic Review of the Therapeutic Role of Hydroxychloroquine in Coronavirus Disease-19 (COVID-19)." Clinicaldrug investigation vol. 40,7 (2020)

4. Protocole de prise en charge des patients des patients atteints de Covid-19 et leurs contacts. Circulaire n²3/DELM/2020 du 24 Mars (2020)

5. Caster, O, Aoki, Y, Gattepaille, L.M. et al. Disproportionality Analysis for Pharmacovigilance Signal Detection in Small Databases or Subsets: 
Recommendations for Limiting False-Positive Associations. Drug Saf 43, 479-487 (2020)

6. WHO-UMC system for causality assessment available

from :https://www.who.int/medicines/areas/quality safety/safety efficacy/WHOcausality assessment

7. Benkirane.R, Soulaymani,R, Khattabi.A, Benabdallah.G, Alj.L, Sefiani.H, Hedna.K, Ouammi.L, Olsson.S and Pal.SN. Assessment of a new instrument for detecting preventable adverse drug reactions. Drug safety.; 38 : 383-393 (2015)

8. MedDRA ${ }^{\circledR}$ term selection:points to consider https://www.meddra.org/ March (2021)

9. Coccia.M The Fishbone diagram to identify, systematize and analyze the sources of general purpose technologies, Project: emerging technologies, R\&D performance of labs, metrics and classifications of innovations, December (2017)

10. Edwards IR, Aronson JK. Adverse drug reactions: definitions, diagnosis, and management. Lancet; 356: 1255-1259. (2000)

11. Koh Y, Yap CW, Li S-C. Development of a combined system for identification and classification of adverse drug reactions: alerts based on ADR Causality and Severity (ABACUS). J Am Med Inform Assoc; 17: 720-722 (2010)

12. Drugs.com. Hydroxychloroquine Sulfate Monograph for Professionals. 2020. Available online:https://www.drugs.com/monograph/hydroxyc hloroquine-sulfate.html

13. Choudhuri, AnirbanHom et al. "The efficacy and safety of hydroxychloroquine (HCQ) in treatment of COVID19 -a systematic review and meta-analysis." Indian journal of medicalmicrobiology vol. 39,2 (2021)

14. Council for International Organizations of Medical Sciences. Practical aspects of signal detection in pharmacovigilance: Report of CIOMS Working Group VIII. Geneva: CIOMs; 2010.
Annexe 1: adapted reporting form

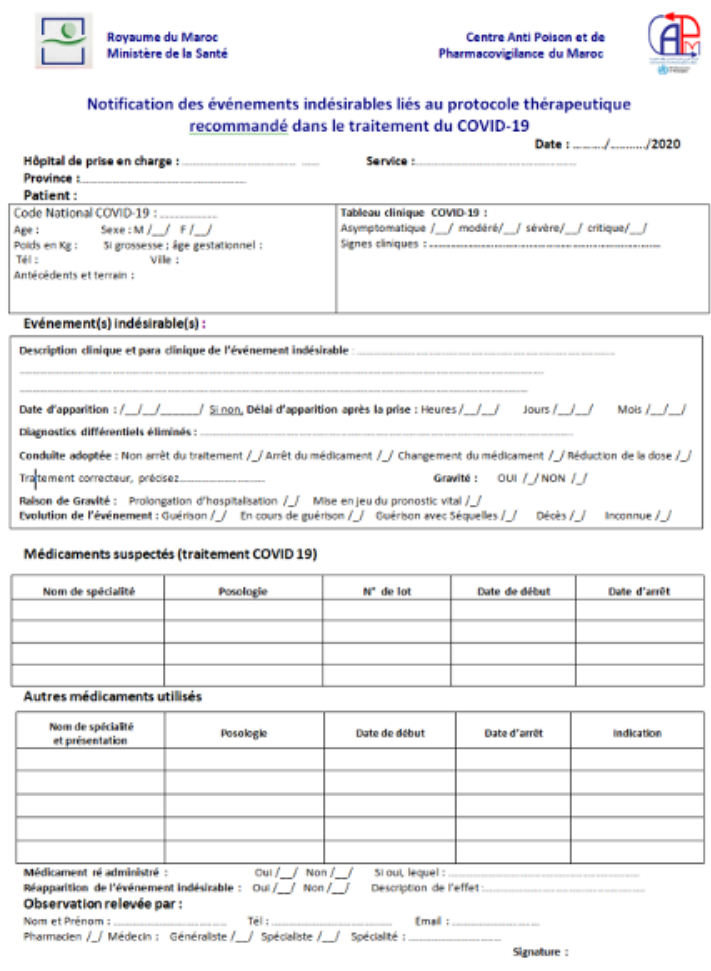

